OPEN ACCESS

Edited by:

Margaret K. Hahn,

University of Toronto, Canada

Reviewed by:

Sarah Tosato,

University of Verona, Italy

Anders Fink-Jensen,

Region Hovedstad

Psychiatry, Denmark

*Correspondence:

Elina J. Reponen

e.j.reponen@medisin.uio.no

Specialty section

This article was submitted to

Schizophrenia

a section of the journal

Frontiers in Psychiatry

Received: 29 October 2020 Accepted: 23 April 2021

Published: 28 May 2021

Citation:

Reponen EJ, Tesli M, Dieset I,

Steen NE, Vedal TSJ, Szabo A,

Werner MCF, Lunding SH,

Johansen IT, Rødevand LN,

Andreassen $O A$ and Ueland $T$ (2021)

Adiponectin Is Related to

Cardiovascular Risk in Severe Mental

Illness Independent of Antipsychotic

Treatment.

Front. Psychiatry 12:623192.

doi: 10.3389/fpsyt.2021.623192

\section{Adiponectin Is Related to Cardiovascular Risk in Severe Mental Illness Independent of Antipsychotic Treatment}

\author{
Elina J. Reponen ${ }^{1 *}$, Martin Tesli ${ }^{1,2}$, Ingrid Dieset ${ }^{1,3}$, Nils Eiel Steen ${ }^{1}$, Trude S. J. Vedal ${ }^{1}$, \\ Attila Szabo ${ }^{1}$, Maren C. F. Werner ${ }^{1}$, Synve H. Lunding ${ }^{1}$, Ingrid T. Johansen ${ }^{1}$, \\ Linn N. Rødevand ${ }^{1}$, Ole A. Andreassen ${ }^{1}$ and Thor Ueland ${ }^{4,5,6}$ \\ ${ }^{1}$ NORMENT, Norwegian Centre for Mental Disorders Research, Oslo University Hospital and University of Oslo, Oslo, \\ Norway, ${ }^{2}$ Department of Mental Disorders, Norwegian Institute of Public Health, Oslo, Norway, ${ }^{3}$ Division of Mental Health \\ and Addiction, Acute Psychiatric Department, Oslo University Hospital, Oslo, Norway, ${ }^{4}$ Institute of Clinical Medicine, \\ University of Oslo, Oslo, Norway, ${ }^{5}$ Research Institute of Internal Medicine, Oslo University Hospital Rikshospitalet, Oslo, \\ Norway, ${ }^{6}$ K.G. Jebsen Thrombosis Research and Expertise Center, University of Tromsø, Tromsø, Norway
}

Background: Schizophrenia (SCZ) and bipolar disorder (BD) are severe mental illnesses (SMI) associated with elevated cardiovascular disease (CVD) risk, including obesity. Leptin and adiponectin are secreted by adipose tissue, with pro- and anti-inflammatory properties, respectively. The second generation antipsychotics (AP) olanzapine, clozapine, and quetiapine have been associated with high leptin levels in SMI. However, the link between inflammatory dysregulation of leptin and adiponectin and CVD risk in SMI, and how this risk is influenced by body mass and AP medication, is still not completely understood. We investigated herein if leptin, adiponectin or their ratio ( $L / A$ ratio) could predict increased CVD risk in SCZ, BD, and in subgroups according to use of antipsychotic (AP) treatment, independent of other cardio-metabolic risk factors.

Methods: We measured fasting plasma levels of leptin and adiponectin, and calculated the L/A ratio in $n=1,092$ patients with $\mathrm{SCZ}$ and $\mathrm{BD}$, in subgroups according to $\mathrm{AP}$ treatment, and in $n=176$ healthy controls $(\mathrm{HC})$. Differences in the levels of adipokines and L/A between groups were examined in multivariate analysis of covariance, and the correlations between adipokines and body mass index (BMI) with linear regression. CVD risk was defined by total cholesterol/high-density lipoprotein (TC/HDL) and triglyceride/HDL (TG/HDL) ratios. The adipokines and L/A ratios ability to discriminate individuals with $\mathrm{TG} / \mathrm{HDL}$ and $\mathrm{TC} / \mathrm{HDL}$ ratios above threshold levels was explored by ROC analysis, and we investigated the possible influence of other cardio-metabolic risk factors on the association in logistic regression analyses.

Results: We observed higher leptin levels and L/A ratios in SMI compared with HC but found no differences in adiponectin. Both adipokines were highly correlated with BMI. The low adiponectin levels showed a fair discrimination in ROC analysis of individuals 
with CVD risk, with AUC between 0.7 and 0.8 for both TC/HDL and TG/HDL, in all groups examined regardless of diagnosis or AP treatment. Adiponectin remained significantly associated with an elevated TC/HDL and TG/HDL ratio in SMI, also after further adjustment with other cardio-metabolic risk factors.

Conclusions: Adiponectin is not dysregulated in SMI but is associated with CVD risk regardless of AP treatment regime.

Keywords: leptin, adiponectin, L/A ratio, CVD risk, antipsychotic treatment, schizophrenia, bipolar disorder

\section{INTRODUCTION}

Schizophrenia (SCZ) and bipolar disorder (BD) are severe mental illnesses (SMI) that are associated with an increased cardiovascular disease (CVD) risk (1). Obesity occurs frequently in patients with SCZ and BD and contributes to the elevated cardiovascular risk (2). The prevalence of elevated body-mass index (BMI) in patients with SCZ and BD is estimated to be 3-5 times higher compared with the general population $(2,3)$.

A pro-inflammatory state of the adipose tissue is supposed to accelerate CVD in obese and overweighed subjects and is characterized by augmented production of inflammatory cytokines (4). Thus, leptin and adiponectin are cytokines primarily secreted by adipose tissue (adipokines) with pro- and anti-inflammatory properties, respectively (5). These proteins may have direct effects on atherogenesis in CVD, and experimental studies have demonstrated that leptin promotes, whereas adiponectin attenuates atherosclerosis (6), although a dual role on endothelial cells has recently been described for adiponectin (7). Furthermore, numerous clinical studies implicate dysregulated leptin and adiponectin levels in the progression of CVD (8-11). Thus, both hyperleptinemia and hypoadiponectinemia have been shown to be independently associated with increased fat tissue and CVD risk in the general population. Due to the opposite metabolic effects of leptin and adiponectin, the leptin/adiponectin ratio (L/A ratio) has been proposed as a useful marker for metabolic disease, and may be more strongly associated with CVD risk than leptin or adiponectin alone $(12,13)$.

Schizophrenia and related disorders are associated with dysregulated adipokine levels (14) and we and other have demonstrated that use of antipsychotic (AP) medication (15), especially the $3 \mathrm{~s}$ generation antipsychotics olanzapine, clozapine, and quetiapine $(16,17)$ may enhance the leptin as well as the L/A ratio (15) in SMI. However, the link between dysregulation of the leptin-adiponectin axis and CVD in SMI, and how this risk is modified by body mass and AP medication, have scarcely been examined. Herein vi investigate whether leptin, adiponectin or their ratio (L/A ratio) could be associated with increased CVD risk in $\mathrm{SCZ}$ and $\mathrm{BD}$, and in subgroups according to $\mathrm{AP}$ treatment, independent of other established cardio-metabolic risk factors.

Our specific aims of this study were 4-fold. Firstly, we evaluate whether the distribution of leptin and adiponectin and their ratio differ between patients with SMI (SCZ or BD) compared to healthy controls (HC), and between subgroups of AP medication, and secondly if this difference is mitigated by
BMI. Thirdly, we investigate if these adipokines or their ratio can discriminate individuals with or without pro-atherogenic lipid ratios above threshold levels, and fourthly, if any association with these lipid ratios is independent or modified by other established cardio-metabolic risk factors: age, sex, BMI, Creactive protein (CRP), insulin resistance (HOMA-IR), smoking, and anti-psychotic, anticonvulsant, and lithium treatment dose (DDD). As a measure of CVD risk we calculated pro-atherogenic lipid ratios total cholesterol/high-density lipoprotein; HDL-c (TC/HDL) and triglyceride/HDL-c (TG/HDL), based on our previously published results (18).

We hypothesize to find significantly higher leptin levels and significantly lower adiponectin levels in SCZ and BD compared to healthy controls, mitigated by BMI. We expect the dysregulated adipokine levels and particularly L/A to independently predict elevated atherogenic lipid ratios in all groups, and in particular in patients using AP treatment, and in patients using olanzapine, clozapine or quetiapine.

\section{METHODS}

\section{Design and Ethics}

This cross-sectional study is a part of the large ongoing Thematically Organized Psychosis (TOP) Study at the Norwegian Centre for Mental Disorders Research (NORMENT). Patients in the TOP study are included from hospitals and outpatient clinics in the Oslo, Trondheim, and Lillehammer regions in Norway. The sample for this current study consists of patients and healthy controls included from year 2002 until 2015, all with fasting blood samples available. Both the patients and the HC have given written informed consent, and the study was approved by the Norwegian Scientific Ethical Committees and the Norwegian Data Protection Agency.

\section{Sample \\ Patients}

In the current study, 701 patients with schizophrenia spectrum disorder and 391 patients with bipolar spectrum disorder were included, with age between 18 and 65 years.

The diagnostic evaluation of the patients was based on the SCID-1 (Structured Clinical Interview in Diagnostic and Statistical Manual of Mental Disorders, 4th Edition (DSM-IV) axis I Disorders) [The inter-investigator diagnostic agreement has previously been evaluated to a satisfying level of $82 \%$, with overall $\kappa=0.77$ (CI 0.60-0.94) (19)]. Symptoms were evaluated using the Positive and Negative Syndrome Scale (PANSS), and 
the Calgary Depression Scale for Schizophrenia (CDSS), and patient medication records and smoking habits were registered.

A diagnosis of schizophrenia spectrum disorder (SCZ) included the diagnoses of schizophrenia, schizoaffective disorder, schizophreniform disorder, and psychotic disorder not otherwise specified, while a diagnosis of bipolar spectrum disorder (BD) included the diagnoses of bipolar I, bipolar II, and bipolar disorder not otherwise specified.

In this study, SMI is defined as the SCZ and BD groups combined. The subgroups according to antipsychotic treatment were defined as follows: those patients with SMI receiving second generation AP treatment with olanzapine, clozapine or quetiapine were the $\mathrm{AP}^{\mathrm{O} / \mathrm{C} / \mathrm{Q}}(n=522)$ group, those receiving other, first or second generation, AP treatment were the AP $(n=$ $269)$ group, and those patients who did not receive antipsychotic treatment were the AP- $(n=301)$ group.

\section{Healthy Controls}

The current study included 176 healthy controls (HC), between 18 and 65 years old. The HC were randomly selected from statistical records (www.ssb.no) in the Oslo region. Exclusion criterion for HC was current or previous SMI in index persons or their family members, assessed with the clinical interview Primary Care Evaluation of Mental Disorders (PRIME MD).

\section{The Exclusion Criteria}

The exclusion criteria for all participants in the study were: on-going infections, C-reactive protein (hs-CRP) $>20 \mathrm{mg} / \mathrm{L}$ of any reason, on-going autoimmune or inflammatory diseases, ongoing cancer, treatment with immune modulating medication of any reason, or insulin levels $<400 \mathrm{pmol} / \mathrm{L}$ (for valid calculation of insulin resistance). As we investigated atherogenic lipid ratios in our study, a diabetic profile or other dysregulated metabolic parameters were not an exclusion criteria.

\section{Body Mass Index}

All participants were weighed on calibrated digital weights under standard conditions, height was measured with standard methods and body mass index (BMI) $\left(\mathrm{kg} / \mathrm{m}^{2}\right)$ calculated.

\section{Defined Daily Dose for Antipsychotics, Anticonvulsants, and Lithium}

Information on the use of prescribed antipsychotics, anticonvulsants, and lithium was assessed by clinical interview and hospital records. "Defined daily dose" (DDD) of the medication was calculated according to the World Health Organization (WHO) principles. For antipsychotic or anticonvulsant medication, we calculated the individual total DDD based on polypharmacy. The DDD is the assumed average maintenance dose per day for a drug used for its main indication in adults and provide a fixed unit of measurement independent of dosage form (http://www.whocc.no/atc_ddd_ index/).

\section{Blood Samples}

Fasting blood samples were collected between 8 am and 11 am for the most participants. Blood samples were drawn into EDTA tubes, stored at room temperature for $45 \mathrm{~min}$ and placed in refrigerator at 4 degrees $C$. They were then transported to the Biobank the following workday, where $2 \times 9 \mathrm{ml}$ EDTA tubes were centrifuged at $1,800 \mathrm{~g}$ for $15 \mathrm{~min}$. Plasma was collected and stored at -80 degrees $\mathrm{C}$ in multiple aliquots (20).

\section{Biochemistry}

Plasma levels of total cholesterol (TC), triglyceride (TG), high-density lipoprotein (HDL-c), and low-density lipoprotein (LDL-c) were measured on an Integra 800 instrument from Roche Diagnostics, according to standard methods. Leptin, adiponectin and C-reactive protein (CRP) were analyzed using standardized platforms from Roche Diagnostics. All analyses were performed at the Department of Medical Biochemistry, Oslo University Hospital.

\section{Insulin Resistance}

Glucose and insulin were analyzed at the Department of Medical Biochemistry, Oslo University Hospital. Glucose levels were analyzed using standardized platforms from Roche Diagnostics. Insulin was analyzed at the Hormone Laboratory by radioimmunoassay (RIA) using standard methods. We estimated insulin resistance using the Homeostasis Model Assessment for Insulin Resistance (HOMA-IR) (21). As the calculation is valid only with insulin levels $<400 \mathrm{pmol} / \mathrm{L}$, participants with higher levels were excluded $(n=11)$.

\section{Cardiovascular Risk}

Cardiovascular risk was estimated calculating established proatherogenic lipid ratios including TG/HDL and TC/HDL, with sex-dependent cut-offs established elsewhere $(22,23)$.

\section{Statistical Analyses}

All statistical analyses were done using the SPSS software package for Windows, version 26.0 (SPSS Chicago. USA). All analyses were two-tailed with a level of significance set at $p<0.05$. All skewed data was log-transformed prior to further analyses. Demographics of the study population were analyzed with analysis of covariance (ANOVA) for continuous variables and chi-square test for independence for categorical variables.

Differences in levels of adipokines, between $\mathrm{HC}$ and diagnostic groups (i.e., SCZ and BD) were analyzed by multivariate analysis of covariance (MANCOVA), adjusting stepwise for age, sex, duration of illness, and BMI. The same analysis model was then repeated for the $\mathrm{AP}, \mathrm{AP}-$, and $\mathrm{AP} / \mathrm{C} / \mathrm{Q}$ subgroups. Further adjustment included mood stabilizers; anticonvulsive, and lithium treatment dose (DDD) as well as duration of AP treatment. We then analyzed correlations with linear regression analysis between BMI and leptin, adiponectin or $\mathrm{L} / \mathrm{A}$ ratio across the studied groups.

To evaluate if adipokines or their ratio could discriminate individuals with or without pro-atherogenic lipid ratios (TG/HDL and TC/HDL) above threshold levels, we then performed receiver operating characteristics (ROC) analysis of leptin, adiponectin, and $\mathrm{L} / \mathrm{A}$ ratio.

Finally, we evaluated the association between adipokine levels and pro-atherogenic risk using logistic regression with different 
adjustment levels to assess if the associations were independent or modified by other cardio-metabolic risk factors in BD, SCZ and HC. The same analysis model was then repeated for the $\mathrm{AP}, \mathrm{AP}-$, and $\mathrm{AP} / \mathrm{C} / \mathrm{Q}$ subgroups. Multivariable adjustment included age, sex, BMI, C-reactive protein (CRP), insulin resistance (HOMA-IR), smoking, anti-psychotic, anticonvulsive, and lithium treatment dose (DDD), duration of AP treatment and duration of illness.

\section{RESULTS}

\section{Sample Characteristics}

The clinical characteristics of the study population according to AP treatment are shown in Table $\mathbf{1}$ and the clinical characteristics according to diagnostic groups are shown in Supplementary Table 1. Patients with SMI were of a similar age compared to $\mathrm{HC}$, but when looking at the diagnostic groups, patients with $\mathrm{BD}$ were older than SCZ and had a longer duration of illness. Patients were less frequently male and of European origin than HC. Patients with SCZ received more often anti-psychotic treatment than BD but less anticonvulsants and lithium. Comparing a range of cardio-metabolic risk factors including HOMA-IR, CRP, BMI and lipids revealed a generally higher burden in patients with SMI was revealed, especially in SCZ compared with HC. Focusing on pro-atherogenic lipid ratios, patients with SMI had a 2- and 3-times higher proportion of individuals with TC/HDL and TG/HDL above threshold limits, respectively. Evaluated within the diagnostic groups, particularly SCZ patients had elevated ratios. These findings are similar to our previously published results on pro-atherogenic lipid ratios in a partly overlapping sample (18).

Evaluation of demographics according to antipsychotic (AP) treatment revealed a lower proportion of male patients that did not receive AP treatment (AP-). In general, patients using AP (including $\mathrm{AP}^{\mathrm{O} / \mathrm{C} / \mathrm{Q}}$ ) had a higher metabolic burden compared with those not receiving AP (both AP- and $\mathrm{HC}$ ). The highest proportion of dysregulated pro-atherogenic lipid ratios (i.e., TC/HDL and TG/HDL) were observed in patients using AP (including $\mathrm{AP}^{\mathrm{O} / \mathrm{C} / \mathrm{Q}}$ ) followed by those not using $\mathrm{AP}$ (AP-), and then HC.

\section{Adipokine and L/A Ratio Levels in SMI and Between AP Subgroups}

As shown in Figure 1A, patients as a whole were characterizled by markedly higher leptin levels compared to HC $(p=0.005)$ in age-, sex-, and duration of illness adjusted analysis. Further, patients using $\mathrm{AP}$ (both $\mathrm{AP}$ and $\mathrm{AP}^{\mathrm{O} / \mathrm{C} / \mathrm{Q}}$ ), had markedly higher leptin levels compared to patients not using AP (AP-), $(p<$ 0.01 for both AP groups vs. AP-) and $\mathrm{HC}(p<0.01$ for both AP groups vs. HC), in analysis with further adjustment for AP treatment duration, and treatment with mood stabilizers (anticonvulsants and lithium). The same pattern of higher leptin levels was observed when evaluating the diagnostic groups SCZ or BD according to AP use (Supplementary Figure 1A).

As shown in Figure 1B and in Supplementary Figure 1B, no significant differences in adiponectin levels was observed between $\mathrm{HC}$ and SMI or according to AP use with similar findings within SCZ and BD.

As shown in Figure 1C and in Supplementary Figure 1C, the $\mathrm{L} / \mathrm{A}$ ratio mirrored leptin levels although the differences between groups were somewhat attenuated. Thus, patients with SMI have an elevated L/A ratio compared to $\mathrm{HC}(p=0.041)$, and AP users had a higher ratio than non-AP users $(p<0.002$ for both AP groups) with a similar pattern, but larger confidence intervals attenuated significance, in diagnostic SCZ and BD groups.

Leptin was correlated with duration of illness $(r=0.11, p<$ $0.001)$ and duration of AP treatment $(r=0.18, p<0.001$. DDD for anticonvulsive therapy correlated modestly with adiponectin levels $(r=0.15, p<0.001)$ and the L/A ratio $(r=-0.09, p=$ 0.004). DDD for lithium correlated modestly with leptin levels $(r=0.11, p<0.001)$ and the L/A ratio $(r=-0.09, p=0.002)$. No other correlation between adipokine levels DDD for lithium and anticonvulsive therapy or duration of illness or AP treatment were detected.

\section{Adipokine Distribution by BMI}

As shown in Figure 1A, leptin and BMI were strongly positively correlated in all groups $(p<0.001)$ and no interaction between $\mathrm{BMI}$ and AP group (i.e., group* BMI) was observed. Accordingly, the marked differences observed in age- and sex-adjusted analysis were largely mitigated by BMI adjustment and no differences in leptin levels between $\mathrm{SMI}, \mathrm{HC}$ or $\mathrm{AP}, \mathrm{AP}$ - and $\mathrm{AP} / \mathrm{C} / \mathrm{Q}$ groups were observed after this adjustment.

As shown in Figure 1B, adiponectin correlated negatively with BMI ( $p<0.001$ for all groups), but correction for BMI did not reveal differences between the studied groups.

As shown in Figure 1C, the L/A ratio was strongly positively correlated with BMI ( $p<0.001$ for all groups) and the differences between diagnostic and between sub-groups according to AP use were diminished following BMI adjustment.

\section{Discriminating Power of Adipokines on Atherogenic Lipid Ratios}

Figure $2 \mathrm{~A}$ shows ROC analysis of leptin, adiponectin, and L/A ratio levels ability to discriminate individuals with or without pro-atherogenic lipid ratios above treshold levels. The leptin levels were poor in identifying patients with elevated proatherogenic as reflected by the area under the curve (AUC) $<$ 0.7 for TC/HDL and TG/HDL across the groups analyzed. The adiponectin levels showed a fair discrimination of individuals with elevated atherogenic ratios. The reciprocal values of the ROC analysis (i.e., inverse) indicated AUC between 0.7 and 0.8 with no major differences in $\mathrm{HC}$, SMI or according to AP treatment. Notably, the best discrimination was observed in the AP- group with an AUC of 0.78 (inverse of 0.22 ). The $\mathrm{L} / \mathrm{A}$ ratio was better than leptin but still poor in identifying individuals with elevated $\mathrm{TC} / \mathrm{HDL}$ ratio (AUC $<0.7$ ) but somewhat better in identifying individuals with an elevated TG/HDL ratio (AUC $>0.7$ ). This pattern was seen across all groups studied but was notably poorer in the AP- group vs. TG/HDL $(\mathrm{AUC}=0.65)$. 
TABLE 1 | Demographics of the study population.

\begin{tabular}{|c|c|c|c|c|c|c|}
\hline & $\mathrm{HC}$ & SMI & AP- & AP & $\mathrm{APO} / \mathrm{C} / \mathrm{Q}$ & Post-hoc \\
\hline $\mathrm{N}$ & 176 & 1092 & 301 & 269 & 522 & \\
\hline Sex (male) & $113(64)$ & $576(53)^{\star \star}$ & $139(46)$ & $141(52)$ & $296(57)$ & $\mathrm{HC}, \mathrm{APO} / \mathrm{C} / \mathrm{Q}>\mathrm{AP}-$ \\
\hline Age & $32 \pm 8$ & $32 \pm 11$ & $33 \pm 12$ & $31 \pm 10$ & $31 \pm 10$ & \\
\hline Ethnicity (European) & $173(98)$ & $893(82)^{\star \star \star}$ & $254(84)$ & $209(78)$ & $430(82)$ & $\mathrm{HC}>\mathrm{AP}-, \mathrm{AP}, \mathrm{APO} / \mathrm{C} / \mathrm{Q}$ \\
\hline Duration of illness, years & N/A & $9.9(8.9)$ & $12.0(9.8)$ & $8.9(8.1)$ & $9.1(8.5)$ & $\mathrm{AP}->\mathrm{AP}, \mathrm{APO} / \mathrm{C} / \mathrm{Q}$ \\
\hline Daily smoking & N/A & $492(46)$ & $120(41)$ & $135(52)$ & $237(46)$ & \\
\hline Statin use & $0(0)$ & $15(1.5)$ & $1(0.3)$ & $9(3.3)$ & $6(1.1)$ & $A P>A P-$ \\
\hline Antipsychotics (DDD) & N/A & $0.88(0.96)$ & $0(0)$ & $0.93(0.69)$ & $1.35(1.01)$ & $\mathrm{APO} / \mathrm{C} / \mathrm{Q}>\mathrm{AP}>\mathrm{AP}-$ \\
\hline Duration of AP treatment, months & N/A & $9.7(24.6)$ & $0(0)$ & $11.6(29.4)$ & $14.2(27.1)$ & \\
\hline Anticonvulsants (DDD) & N/A & $0.14(0.35)$ & $0.17(0.39)$ & $0.10(0.29)$ & $0.14(0.35)$ & $A P->A P$ \\
\hline Lithium (DDD) & N/A & $0.08(0.30)$ & $0.09(0.33)$ & $0.05(0.22)$ & $0.09(0.32)$ & $\mathrm{APO} / \mathrm{C} / \mathrm{Q}>\mathrm{AP}$ \\
\hline \multicolumn{7}{|l|}{ Cardiometabolic Risk Factors } \\
\hline HOMA-IR & $1.2 \pm 0.7$ & $1.7 \pm 1.0^{\star \star \star}$ & $1.4 \pm 0.8$ & $1.7 \pm 0.9$ & $1.8 \pm 1.1$ & $\mathrm{APO} / \mathrm{C} / \mathrm{Q}, \mathrm{AP}>\mathrm{AP}-, \mathrm{HC}$ \\
\hline $\mathrm{CRP}(\mathrm{mg} / \mathrm{L})$ & $1.6 \pm 2.2$ & $2.3 \pm 2.8^{\star \star \star}$ & $2.0 \pm 2.5$ & $2.6 \pm 3.3$ & $2.4 \pm 2.7$ & $\mathrm{APO} / \mathrm{C} / \mathrm{Q}, \mathrm{AP}>\mathrm{AP}->\mathrm{HC}$ \\
\hline BMl & $24.2 \pm 3.6$ & $25.8 \pm 4.6^{\star \star \star \star}$ & $24.8 \pm 4.3$ & $26.3 \pm 5.0$ & $26.0 \pm 4.5$ & $\mathrm{APO} / \mathrm{C} / \mathrm{Q}, \mathrm{AP}>\mathrm{AP}-, \mathrm{HC}$ \\
\hline HDL-c(mmol/L) & $1.47 \pm 0.39$ & $1.38 \pm 0.43^{\star}$ & $1.48 \pm 0.45$ & $1.35 \pm 0.41$ & $1.34 \pm 0.42$ & $\mathrm{HC}, \mathrm{AP}->\mathrm{AP}, \mathrm{APO} / \mathrm{C} / \mathrm{Q}$ \\
\hline LDL-c (mmol/L) & $2.94 \pm 0.88$ & $3.13 \pm 0.95^{\star}$ & $3.01 \pm 0.90$ & $3.05 \pm 0.89$ & $3.24 \pm 0.99$ & APO/C/Q> HC,AP-,AP \\
\hline Total-c (mmol/L) & $4.70 \pm 0.95$ & $5.07 \pm 1.07^{\star \star \star}$ & $4.97 \pm 1.07$ & $5.03(1.02)$ & $5.16 \pm 1.09$ & $\mathrm{AP}-, \mathrm{AP}, \mathrm{APO} / \mathrm{C} / \mathrm{Q}>\mathrm{HC}$ \\
\hline Triglycerides (mmol/L) & $1.07 \pm 0.78$ & $1.39 \pm 0.99^{\star \star \star}$ & $1.19 \pm 0.71$ & $1.48 \pm 1.19$ & $1.45 \pm 1.00$ & $\mathrm{AP}, \mathrm{APO} / \mathrm{C} / \mathrm{Q}>\mathrm{HC}, \mathrm{AP}-$ \\
\hline Total-c/HDL-c & $21(12)$ & $257(24)$ & $50(17)$ & $65(24)$ & $142(27)$ & $\mathrm{APO} / \mathrm{C} / \mathrm{Q}, \mathrm{AP}>\mathrm{AP}->\mathrm{HC}$ \\
\hline Triglycerides/HDL-c & $17(10)$ & $301(28)$ & 58 (19) & $85(32)$ & $158(30)$ & $\mathrm{APO} / \mathrm{C} / \mathrm{Q}, \mathrm{AP}>\mathrm{AP}->\mathrm{HC}$ \\
\hline \multicolumn{7}{|l|}{ Symptom scores } \\
\hline PANSS total & N/A & $0.22 \pm 0.13$ & $0.22 \pm 0.12$ & $0.24 \pm 0.12$ & $0.22 \pm 0.13$ & \\
\hline CDSS total & $\mathrm{N} / \mathrm{A}$ & $5.3 \pm 4.7$ & $5.7 \pm 4.4$ & $5.5 \pm 5.2$ & $5.0 \pm 4.6$ & \\
\hline
\end{tabular}

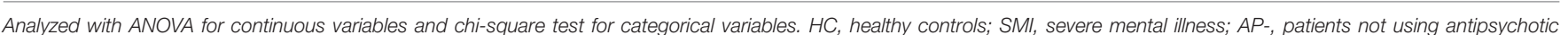

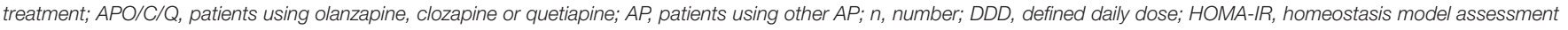

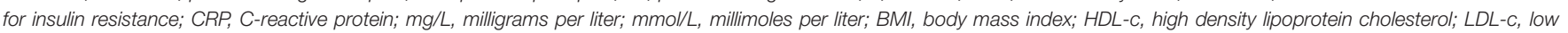

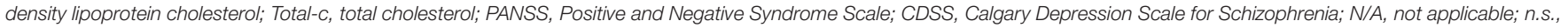
not significant, ${ }^{*} p<0.05^{* *} p<0.01^{\star \star *} p<0.001$ vs. HC.

\section{Association Between Adipokine Levels and Atherogenic Lipid Ratios}

We next evaluated the association between adipokine levels and elevated pro-atherogenic ratios using logistic regression with different adjustment levels to assess if the associations were independent or modified by other cardio-metabolic risk factors, mood stabilizers (anticonvulsants and lithium), duration of illness, and duration of AP treatment. Odds ratios (OR) were for these analyses based on log-transformed standardized values, and represent a one standard deviation (SD) increase in the analyzed marker. Based on the poor performance of leptin in the ROC analysis, we focused on adiponectin and the L/A ratio. The results of these analyses are presented in Figure $2 \mathbf{B}$ and Supplementary Tables 2, 3. As presented in Figure 2B, the OR for adiponectin and the L/A ratio was largely comparable in age- and sex-adjusted analysis in identifying individuals that had an elevated TC/HDL or TG/HDL ratio with overlapping CI's between all groups. The numeric for this analysis as well as for the diagnostic groups and subgroups according to AP use are presented in Supplementary Tables 2, 3.

The OR's were somewhat attenuated upon addition of BMI but both the L/A ratio and in particular adiponectin, remained significantly associated with an elevated TC/HDL and TG/HDL ratio, also after further adjustment with other cardio-metabolic risk factors (i.e., CRP, smoking, HOMA-IR, DDD for AP), mood stabilizers, duration of illness, and duration of AP treatment to the models. The lack of significance in the HC population is largely due the lower frequency of dyslipidemia in this group as reflected by large CI's but comparable point estimates (OR) were observed in HC compared to the other groups. Altogether, the L/A ratio and in particular adiponectin were independently associated with atherogenic risk in all diagnostic groups and treatment modalities.

\section{DISCUSSION}

We investigated dysregulation of the prototypical adipokines leptin, adiponectin, and leptin/adiponectin ratio in a large clinical sample of patients with SMI, associations with elevated atherogenic lipid ratios, and whether these associations were dependent on antipsychotic treatment. We found that (i) patients with SMI displayed markedly elevated leptin levels and L/A ratio, with particularly high levels observed in AP users while adiponectin was comparable in all groups, that (ii) all markers correlated strongly with BMI with similar associations in all diagnostic groups and AP treatment regimes, and that (iii) low adiponectin and a high L/A ratio were associated with elevated lipid ratios, and (iv) the association with elevated lipid ratios was independent of other cardio-metabolic risk factors; $\mathrm{BMI}, \mathrm{CRP}$, insulin resistance and smoking, with comparable 


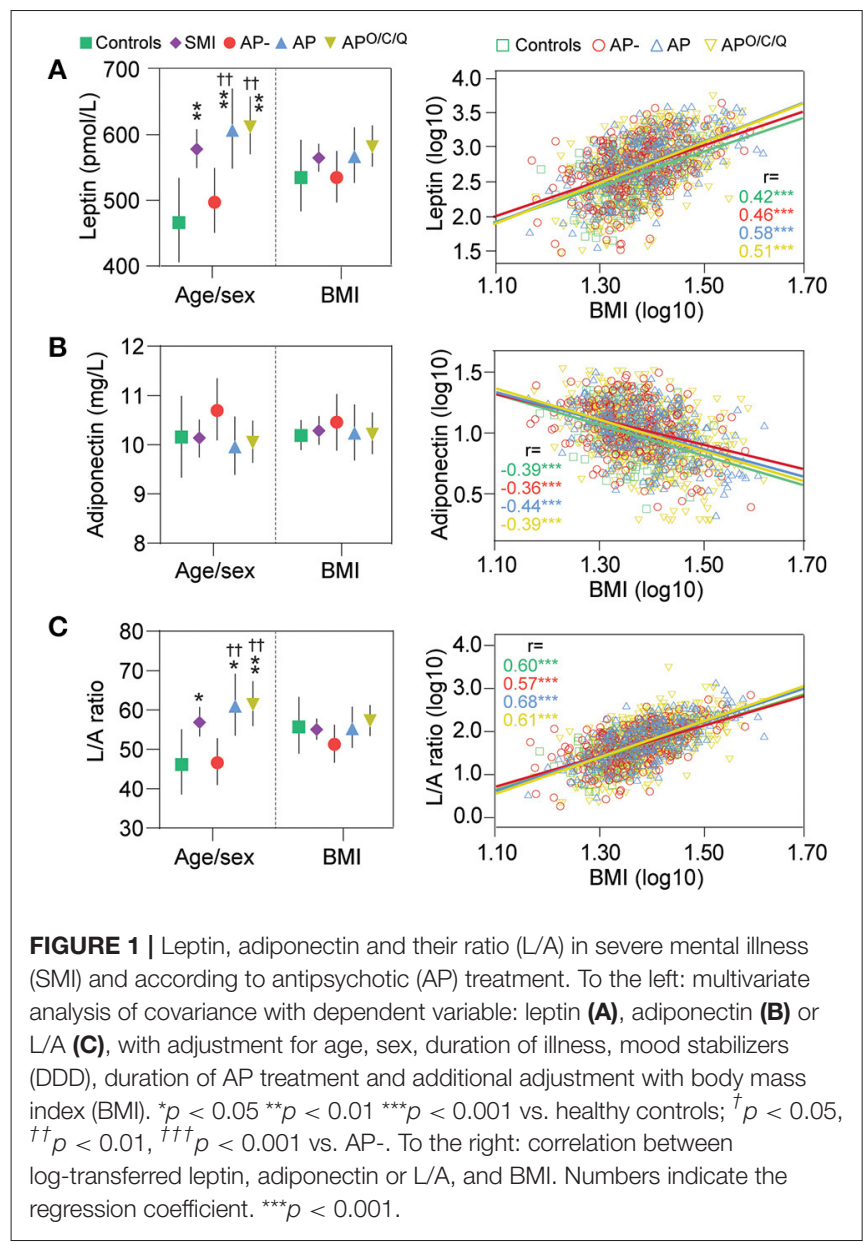

associations with risk according to AP use. Altogether, elevated leptin in SMI was largely driven by BMI and not strongly associated with elevated lipid ratios. In contrast, adiponectin was not dysregulated, however, low levels were strongly and independently associated with the elevated lipid ratios. We were unable to detect that any adverse effect of AP treatment on elevated lipid ratios is conveyed by these adipokines as reflected by their circulating levels.

Obesity and overweight are frequent in SMI and are associated with increased leptin levels, closely correlated with BMI and AP treatment, as confirmed in the present study and shown previously in numerous other studies $(14,24)$. Increased fat mass is accompanied by infiltration of various myeloid immune cells (such as neutrophils, monocytes and macrophages) in adipose tissue, and altered secretion of adipokines including reduced expression of the insulin sensitizing adiponectin (2527). Furthermore, AP treatment has been shown to promote monocyte infiltration, macrophage effector functions and inflammation in adipose tissue in experimental studies (28-30), as well as to regulate adiponectin expression and secretion (30). Thus, dysregulated adiponectin levels could be expected to be particularly low in AP treatment users. However, in line with recent meta-analyses, adiponectin levels were similar in SMI and HC $(31,32)$. Except for somewhat higher adiponectin levels in

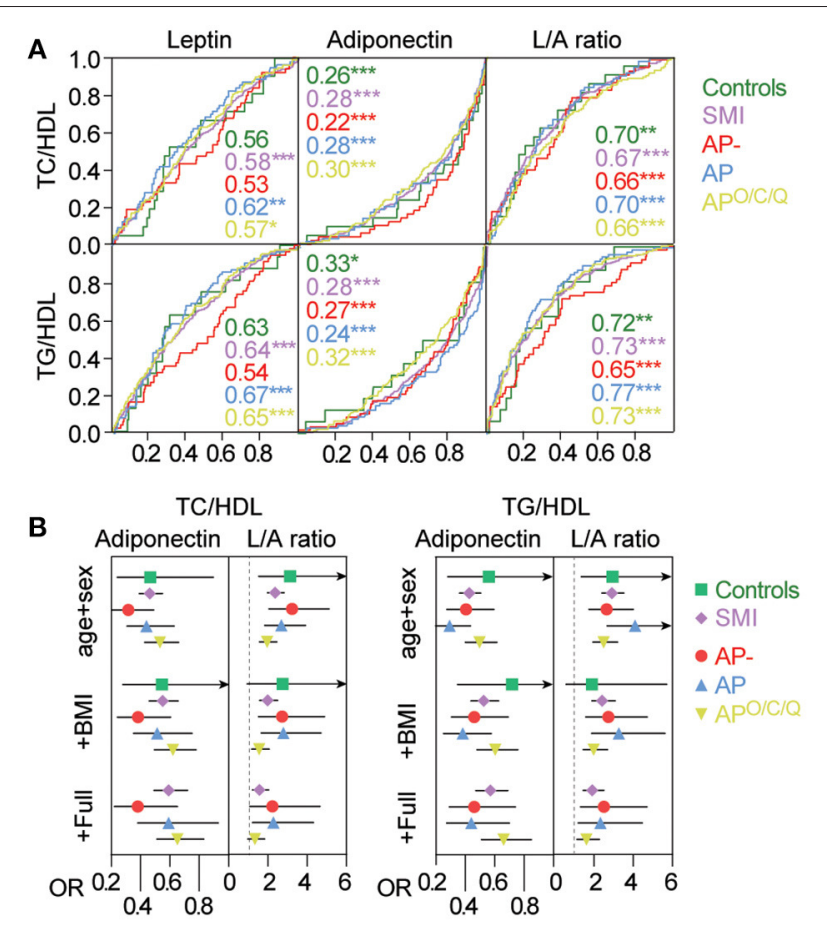

FIGURE 2 | Association between Adiponectin and the L/A ratio and atherogenic risk. (A) Association between Adiponectin or the L/A ratio, and having a TC/HDL or TG/HDL above threshold levels as assessed by the area under the receiver-operating characteristics curve (ROC). Numbers indicate the AUC in the different groups. ${ }^{*} p<0.05^{* \star} p<0.01{ }^{* \star *} p<0.001$. (B) Association between adiponectin and $L / A$ levels and cardiovascular risk (TC/HDL, TG/HDL) using logistic regression with adjustment levels for a. Age and sex b. a+BMl c. b+C-reactive protein (CRP), insulin resistance (HOMA-IR), smoking, and anti-psychotic treatment dose (DDD), duration of illness, mood stabilizers (DDD), duration of AP treatment. Odds ratios (OR) are expressed as log-transformed per SD change in marker. Odds ratios with 95\% Confidence interval are given in Supplementary Tables 2, 3.

AP-, we did not find the levels to be significantly modified by AP use. While several studies report low levels of adiponectin with AP use, and in particular in patients receiving second generation AP treatment $(30,32)$, few of these studies compare with $\mathrm{HC}$ which in our study showed similar levels as in SMI. The differences in L/A ratio between SMI and HC in our study largely reflect leptin levels, since no major dysregulation in adiponectin was detected. Importantly, leptin and adiponectin were closely correlated with BMI in all diagnostic and treatment groups, with comparable regression coefficients and no interactions between group and BMI, suggesting similar production of these adipokines per unit change in BMI. This argues against a more inflamed adipose tissue in SMI, where augmented or antagonized secretion of leptin and adiponectin would be expected, although regional fat distribution would be more informative in this regard.

Obesity in patients with SCZ and BD may contribute to elevated CVD risk, which may be further augmented by AP treatment use (1). Indeed, atherogenic lipid ratios were markedly enhanced as previously shown (18), especially in SCZ and in AP users in our study. Leptin and adiponectin could contribute 
to enhanced CVD risk through metabolic pathways related to obesity and insulin resistance, but could have independent effects as well. Adiponectin has been shown to directly affect HDL and TG metabolism, independent of fat mass, insulin resistance and dyslipidemia $(33,34)$ in non-psychiatric patients, and may modulate signaling pathways in response to inflammatory stimuli in several cell types (35). Thus, adiponectin seems to diminish the inflammatory response in endothelial cells to mechanical injury (36) and modulated macrophages to acquire an antiinflammatory phenotype and inhibited foam cell transformation (37). Adiponectin has recently been demonstrated to have two forms with opposing actions in endothelial cells (7). However, in line with a protective anti-inflammatory role, we found an inverse association between adiponectin and atherogenic lipid ratios in SMI patients. Furthermore, the association with lipid ratios was markedly stronger than for leptin, and persisted following full adjustment for important cardio-metabolic factors.

Focusing on non-psychiatric patients with metabolic disturbances, numerous clinical studies have shown that dysregulated leptin and adiponectin levels $(38,39)$ are strongly associated with dyslipidemia. However, as in the present study, studies on these adipokines and CVD are often cross-sectional using surrogate endpoints for CVD such as lipid ratios (40) or coronary artery calcium (41). There are fewer prospective studies evaluating the association between these adipokines and incident CVD or CVD related outcomes in patients with metabolic distrubances. Shanker et al. demonstrated that low adiponectin and high leptin were associated with incident events in patients with coronary artery disease (42). Low adiponectin was associated with future coronary heart disease in type 2 diabetes (43) as well as CV mortality (44). In contrast, high adiponectin was associated with cardiovascular events in patients with hypertension (45), and in older adults (46). Thus, the association between these adipokines and incident CVD or outcomes seem to depend on the degree of metabolic disturbances.

Evaluating diagnostic groups revealed overall stronger associations in $\mathrm{BD}$ compared to SCZ. Thus, despite quite similar levels, a unit decrease of adiponectin in $\mathrm{BD}$ was associated with a larger risk of having an atherogenic lipid profile than SCZ possibly indicating a more anti-atherogenic effect of adiponectin in BD. As we measured total adiponectin, evaluation of high molecular weight (HMW) adiponectin could have given different results (47). Possibly, different distribution of sub-fractions could also explain the stronger association with CVD risk in $\mathrm{BD}$ but we were unable to find any studies evaluating this. Adipose tissue has been shown to sense and respond to emotional stress through peroxisome-profilator activated receptor $\gamma(\operatorname{PPAR} \gamma)$-adiponectin interactions (48), that are also linked to immunometabolic regulation and systemic inflammation (49), but future studies are needed to evaluate how or if this is relevant in BD.

Increased risk of adverse cardiac events has been associated with AP use, and in particular second generation AP including olanzapine, clozapine, and quetiapine (50). However, we did not observe any clear differences in pro-atherogenic lipid ratios associated with low adiponectin or high L/A ratio, that were dependent on AP use. We found a lower risk of having elevated lipid ratios per unit decrease in adiponectin in users of these $3 \mathrm{~s}$ generation AP, arguing against any adverse effects of these drugs acting through the adiponectin signaling pathway, at least as reflected by circulating levels in a cross-sectional setting. Adverse effects of AP could still be mediated by other pathways, both inflammatory [e.g., activated leukocytes in adipose tissue enhancing inflammation (4)], and non-inflammatory [e.g., effects on lipogenesis and lipolysis (51)].

\section{LIMITATIONS}

Since fasting status effects the levels of adipokines and other confounding cardiovascular variables, a non-fasting status was an exclusion criteria for this study, thus excluding 520 non-fasting available healthy controls, limiting the size of the $\mathrm{HC}$ population. This gave different group sizes of $\mathrm{HC}$ vs. SMI. We also excluded 60 non-fasting BD and 158 non-fasting SCZ patients. The patients with a lower function level can find it challenging to fast over night. Many participants, both controls and patients, have busy everyday lives and were not able to schedule blood sampling in the morning. The non-fasting participants are included in other studies in the overall TOP-study.

For the $\mathrm{HC}$ in our study smoking status is not available. Although the effect of smoking on inflammation is less documented, the effect of smoking on CVD risk is known. Therefore, since we had smoking status in our patients, we were able to adjust for smoking when evaluating the association between adiponectin and L/A ratio and CVD risk in logistic regression analysis.

Patients with major depression with psychotic symptoms were not included in this study due to small sample size and thus limited statistical power.

We were unable to investigate the possible effect of lipid lowering medication (statins) on CVD risk as only 15 patients in our sample were using these agents. Finally, the design of this study is cross-sectional, limiting our ability to conclude on causality of the associations shown.

Furthermore, as our study is associative by nature, causeeffect relationships would have to be shown in a prospective controlled study.

\section{STRENGTHS AND CLINICAL IMPLICATIONS}

To our knowledge, this is to date the largest study evaluating dysregulation of leptin, adiponectin, and leptin/adiponectin ratio in patients with SMI. The patient population was wellcharacterized allowing us to adjust our regression models with relevant cardio-metabolic risk factors as well as use of other mood stabilizers and duration of both illness and AP treatment.

As lifespans in people with SMI are markedly reduced frequently due to CVD, there is a need for treatment options that target modifiable metabolic risk factors. Circulating adiponectin represents a modifiable risk factor that can be efficiently targeted by lifestyle modifications, mainly weight loss and dietary changes (52). Thus, low adiponectin could be used to identify and monitor patients that could benefit from such modifications as well as in increasing awareness of increased CVD risk in these individuals. 


\section{CONCLUSION}

In a large clinical sample of patients with SMI we show that adiponectin is not dysregulated in patients compared to HC, but low levels of adiponectin are associated with enhanced CVD risk regardless of AP treatment regime. Our findings support an antiatherogenic role for adiponectin and suggest it could be further evaluated in novel CVD risk prediction strategies in SMI.

\section{DATA AVAILABILITY STATEMENT}

The datasets presented in this article are not readily available because sharing of data to external parties has not been approved by the ethics committee. Requests to access the datasets should be directed to e.j.reponen@medisin.uio.no.

\section{ETHICS STATEMENT}

The studies involving human participants were reviewed and approved by Regional committees for medical and health research ethics, East Norway (REK 1). The patients/participants provided their written informed consent to participate in this study.

\section{AUTHOR CONTRIBUTIONS}

ER and TU contributed to data collection, literature search, study design, statistical analysis, and manuscript editing. MT

\section{REFERENCES}

1. Penninx BWJH, Lange SMM. Metabolic syndrome in psychiatric patients: overview, mechanisms, and implications. Dial Clin Neurosci. (2018) 20:6373. doi: 10.31887/DCNS.2018.20.1/bpenninx

2. Strassnig M, Kotov R, Cornaccio D, Fochtmann L, Harvey PD, Bromet EJ. Twenty-year progression of body mass index in a county-wide cohort of people with schizophrenia and bipolar disorder identified at their first episode of psychosis. Bipol Disord. (2017) 19:336-43. doi: 10.1111/bdi.12505

3. Pillinger T, McCutcheon RA, Vano L, Mizuno Y, Arumuham A, Hindley G, et al. Comparative effects of 18 antipsychotics on metabolic function in patients with schizophrenia, predictors of metabolic dysregulation, and association with psychopathology: a systematic review and network meta-analysis. Lancet Psychiatry. (2020) 7:64-77. doi: 10.1016/S2215-0366(19)30416-X

4. Liu R, Nikolajczyk BS. Tissue immune cells fuel obesity-associated inflammation in adipose tissue and beyond. Front Immunol. (2019) 10:1587. doi: 10.3389/fimmu.2019.01587

5. Stern JH, Rutkowski JM, Scherer PE. Adiponectin, leptin, and fatty acids in the maintenance of metabolic homeostasis through adipose tissue crosstalk. Cell Metabol. (2016) 23:770-84. doi: 10.1016/j.cmet.2016.04.011

6. Zhang T, Yang P, Li T, Gao J, Zhang Y. Leptin expression in human epicardial adipose tissue is associated with local coronary atherosclerosis. Med Sci Monitor. (2019) 25:9913-22. doi: 10.12659/MSM.918390

7. Adya R, Tan BK, Randeva HS. Differential effects of leptin and adiponectin in endothelial angiogenesis. J Diab Res. (2015) 2015:648239. doi: 10.1155/2015/648239

8. Spranger J, Kroke A, Möhlig M, Bergmann MM, Ristow M, Boeing H, et al. Adiponectin and protection against type 2 diabetes mellitus. Lancet. (2003) 361:226-8. doi: 10.1016/S0140-6736(03)12255-6

9. Iwashima Y, Katsuya T, Ishikawa K, Ouchi N, Ohishi M, Sugimoto K, et al. Hypoadiponectinemia is an independent risk factor for hypertension. Hypertension. (2004) 43:1318-23. doi: 10.1161/01.HYP.0000129281.03801.4b contributed to data collection, literature search, statistical analysis, and manuscript editing. ID and TV contributed to data collection, literature search, and manuscript editing. NS, MW, SL, IJ, LR, and OA contributed to data collection and manuscript editing. AS contributed to literature search and manuscript editing. All authors contributed to the article and approved the submitted version.

\section{FUNDING}

This work was supported by the Research Council of Norway (grant numbers \#213837, \#223273, \#217776) and the SouthEastern (grant numbers \#2017-112, \#2016-064) and Western (grant number \#91141) Norway Regional Health Authorities and the KG Jebsen Stiftelsen (grant number SKGJ-2011-36).

\section{ACKNOWLEDGMENTS}

The authors would like to thank the participants of the study for their contribution. We also wish to thank the Department of Medical Biochemistry, Oslo University Hospital, and professor Karin Toska and Kari Julien for their contribution.

\section{SUPPLEMENTARY MATERIAL}

The Supplementary Material for this article can be found online at: https://www.frontiersin.org/articles/10.3389/fpsyt. 2021.623192/full\#supplementary-material

10. Laughlin GA, Barrett-Connor E, May S, Langenberg C. Association of adiponectin with coronary heart disease and mortality: the rancho bernardo study. Am J Epidemiol. (2007) 165:164-74. doi: 10.1093/aje/ kwk001

11. Sattar N, Wannamethee G, Sarwar N, Chernova J, Lawlor DA, Kelly A, et al. Leptin and coronary heart disease: prospective study and systematic review. J Am Coll Cardiol. (2009) 53:167-75. doi: 10.1016/j.jacc.2008.09.035

12. Larsen MA, Isaksen VT, Moen OS, Wilsgaard L, Remijn M, Paulssen EJ, et al. Leptin to adiponectin ratio - a surrogate biomarker for early detection of metabolic disturbances in obesity. Nutr Metabo Cardiov Dis. (2018) 28:111421. doi: 10.1016/j.numecd.2018.06.020

13. Chen VC, Chen CH, Chiu YH, Lin TY, Li FC, Lu ML. Leptin/Adiponectin ratio as a potential biomarker for metabolic syndrome in patients with schizophrenia. Psychoneuroendocrinology. (2018) 92:34-40. doi: 10.1016/j.psyneuen.2018.03.021

14. Stubbs B, Wang AK, Vancampfort D, Miller BJ. Are leptin levels increased among people with schizophrenia versus controls? A systematic review and comparative meta-analysis. Psychoneuroendocrinology. (2016) 63:14454. doi: 10.1016/j.psyneuen.2015.09.026

15. Vedal TSJ, Steen NE, Birkeland KI, Dieset I, Reponen EJ, Laskemoen JF, et al. Adipokine levels are associated with insulin resistance in antipsychotics users independently of BMI. Psychoneuroendocrinology. (2019) 103:8795. doi: 10.1016/j.psyneuen.2019.01.001

16. Ragguett RM, Hahn M, Messina G, Chieffi S, Monda M, De Luca V. Association between antipsychotic treatment and leptin levels across multiple psychiatric populations: an updated meta-analysis. Human Psychopharmacol. (2017) 32:e2631. doi: 10.1002/hup.2631

17. Potvin S, Zhornitsky S, Stip E. Antipsychotic-induced changes in blood levels of leptin in schizophrenia: a meta-analysis. Can J Psychiatry. (2015) 60(3 Suppl 2):S26-34.

18. Reponen EJ, Dieset I, Tesli M, Mørch RH, Aas M, Vedal TSJ, et al. Atherogenic lipid ratios related to myeloperoxidase and c-reactive 
protein levels in psychotic disorders. Front Psychiatry. 11:672. doi: 10.3389/fpsyt.2020.00672

19. Ringen PA, Melle I, Birkenaes AB, Engh JA, Faerden A, Vaskinn A, et al. The level of illicit drug use is related to symptoms and premorbid functioning in severe mental illness. Acta Psychiatrica Scandinavica. (2008) 118:297304. doi: 10.1111/j.1600-0447.2008.01244.x

20. Hoseth EZ. Aberrant TNF and Notch Signaling Pathways in Schizophrenia and Bipolar Disorder. Oslo: University of Oslo, Faculty of Medicine (2018).

21. Wallace TM, Levy JC, Matthews DR. Use and abuse of HOMA modeling. Diab Care. (2004) 27:1487-95. doi: 10.2337/diacare.27.6.1487

22. Millan J, Pinto X, Munoz A, Zuniga M, Rubies-Prat J, Pallardo LF, et al. Lipoprotein ratios: physiological significance and clinical usefulness in cardiovascular prevention. Vascular Health Risk Manag. (2009) 5:75765. doi: 10.2147/VHRM.S6269

23. Salazar MR, Carbajal HA, Espeche WG, Leiva Sisnieguez CE, Balbin E, Dulbecco CA, et al. Relation among the plasma triglyceride/high-density lipoprotein cholesterol concentration ratio, insulin resistance, and associated cardio-metabolic risk factors in men and women. Am J Cardiol. (2012) 109:1749-53. doi: 10.1016/j.amjcard.2012.02.016

24. Fernandes BS, Dash S, Jacka F, Dodd S, Carvalho AF, Köhler CA, et al. Leptin in bipolar disorder: a systematic review and meta-analysis. Eur Psychiatry. (2016) 35:1-7. doi: 10.1016/j.eurpsy.2016.02.003

25. Galic S, Oakhill JS, Steinberg GR. Adipose tissue as an endocrine organ. Mol Cell Endocrinol. (2010) 316:129-39. doi: 10.1016/j.mce.2009.08.018

26. Zeyda M, Stulnig TM. Adipose tissue macrophages. Immunol Lett. (2007) 112:61-7. doi: 10.1016/j.imlet.2007.07.003

27. Trim W, Turner JE, Thompson D. Parallels in immunometabolic adipose tissue dysfunction with ageing and obesity. Front Immunol. (2018) 9:169. doi: 10.3389/fimmu.2018.00169

28. Zhang Q, He M, Deng C, Wang H, Huang XF. Effects of olanzapine on the elevation of macrophage infiltration and pro-inflammatory cytokine expression in female rats. J Psychopharmacol. (2014) 28:1161-9. doi: 10.1177/0269881114555250

29. Davey KJ, O'Mahony SM, Schellekens H, O'Sullivan O, Bienenstock J, Cotter PD, et al. Gender-dependent consequences of chronic olanzapine in the rat: effects on body weight, inflammatory, metabolic and microbiota parameters. Psychopharmacology. (2012) 221:155-69. doi: 10.1007/s00213-011-2555-2

30. Ferreira V, Grajales D, Valverde ÁM. Adipose tissue as a target for secondgeneration (atypical) antipsychotics: a molecular view. Biochim Biophys Acta Mol Cell Biol Lipids. (2020) 1865:158534. doi: 10.1016/j.bbalip.2019.158534

31. Misiak B, Stramecki F, Kasznia J, Lis M, Stańczykiewicz B. Adiponectin levels in patients with bipolar disorder: a systematic review and meta-analysis. Psychoneuroendocrinology. (2019) 104:74-9. doi: 10.1016/j.psyneuen.2019.02.019

32. Bartoli F, Lax A, Crocamo C, Clerici M, Carrà G. Plasma adiponectin levels in schizophrenia and role of second-generation antipsychotics: a meta-analysis. Psychoneuroendocrinology. (2015) 56:179-89. doi: 10.1016/j.psyneuen.2015.03.012

33. Vergès B, Petit JM, Duvillard L, Dautin G, Florentin E, Galland $\mathrm{F}$, et al. Adiponectin is an important determinant of apoAI catabolism. Arterioscler Thromb Vasc Biol. (2006) 26:13649. doi: 10.1161/01.ATV.0000219611.50066.bd

34. Chan DC, Watts GF, Ng TW, Uchida Y, Sakai N, Yamashita S, et al. Adiponectin and other adipocytokines as predictors of markers of triglyceride-rich lipoprotein metabolism. Clin Chem. (2005) 51:57885. doi: $10.1373 /$ clinchem. 2004.045120

35. Ouchi N, Walsh K. Adiponectin as an anti-inflammatory factor. Clin Chim Acta. (2007) 380:24-30. doi: 10.1016/j.cca.2007.01.026

36. Fisman EZ, Tenenbaum A. Adiponectin: a manifold therapeutic target for metabolic syndrome, diabetes, and coronary disease? Cardiov Diabetol. (2014) 13:103. doi: $10.1186 / 1475-2840-13-103$

37. Kumada M, Kihara S, Ouchi N, Kobayashi H, Okamoto Y, Ohashi K, et al. Adiponectin specifically increased tissue inhibitor of metalloproteinase1 through interleukin-10 expression in human macrophages. Circulation. (2004) 109:2046-9. doi: 10.1161/01.CIR.0000127953.98131.ED

38. López-Jaramillo P, Gómez-Arbeláez D, López-López J, López-López C, Martínez-Ortega J, Gómez-Rodríguez A, et al. The role of leptin/adiponectin ratio in metabolic syndrome and diabetes. Horm Mol Biol Clin Investig. (2014) 18:37-45. doi: 10.1515/hmbci-2013-0053

39. Kumari R, Kumar S, Kant R. An update on metabolic syndrome: metabolic risk markers and adipokines in the development of metabolic syndrome. Diabetes Metab Syndr. (2019) 13:2409-17. doi: 10.1016/j.dsx.2019.06.005

40. Hyun YJ, Koh SJ, Chae JS, Kim JY, Kim OY, Lim HH, et al. Atherogenecity of LDL and unfavorable adipokine profile in metabolically obese, normal-weight woman. Obesity. (2008) 16:784-9. doi: 10.1038/oby.2007.127

41. Larsen BA, Laughlin GA, Cummins K, Barrett-Connor E, Wassel CL. Adipokines and severity and progression of coronary artery calcium: findings from the rancho bernardo study. Atherosclerosis. (2017) 265:16. doi: 10.1016/j.atherosclerosis.2017.07.022

42. Shanker J, Rao VS, Ravindran V, Dhanalakshmi B, Hebbagodi S, Kakkar VV. Relationship of adiponectin and leptin to coronary artery disease, classical cardiovascular risk factors and atherothrombotic biomarkers in the IARS cohort. Thromb Haemo. (2012) 107:769-80. doi: 10.1160/TH12-04-0263

43. Peters KE, Davis WA, Beilby J, Hung J, Bruce DG, Davis TME. The relationship between circulating adiponectin, ADIPOQ variants and incident cardiovascular disease in type 2 diabetes: the fremantle diabetes study. Diabetes Res Clin Pract. (2018) 143:62-70. doi: 10.1016/j.diabres.2018.06.005

44. Liu G, Ding M, Chiuve SE, Rimm EB, Franks PW, Meigs JB, et al. Plasma levels of fatty acid-binding protein 4 , retinol-binding protein 4 , high-molecularweight adiponectin, and cardiovascular mortality among men with type 2 diabetes: a 22-year prospective study. Arterioscler Thromb Vasc Biol. (2016) 36:2259-67. doi: 10.1161/ATVBAHA.116.308320

45. Kim-Mitsuyama S, Soejima H, Yasuda O, Node K, Jinnouchi H, Yamamoto E, et al. Total adiponectin is associated with incident cardiovascular and renal events in treated hypertensive patients: subanalysis of the ATTEMPT-CVD randomized trial. Sci Rep. (2019) 9:16589-9. doi: 10.1038/s41598-019-52977-x

46. Kizer JR, Barzilay JI, Kuller LH, Gottdiener JS. Adiponectin and risk of coronary heart disease in older men and women. J Clin Endocrinol Metab. (2008) 93:3357-64. doi: 10.1210/jc.2008-0640

47. Mangge H, Almer G, Haj-Yahya S, Pilz S, Gasser R, Möller R, et al. Preatherosclerosis and adiponectin subfractions in obese adolescents. Obesity. (2008) 16:2578-84. doi: 10.1038/oby.2008.439

48. Guo M, Li C, Lei Y, Xu S, Zhao D, Lu XY. Role of the adipose PPAR $\gamma$ adiponectin axis in susceptibility to stress and depression/anxiety-related behaviors. Mol Psychiatry. (2017) 22:1056-68. doi: 10.1038/mp.2016.225

49. Guilherme A, Virbasius JV, Puri V, Czech MP. Adipocyte dysfunctions linking obesity to insulin resistance and type 2 diabetes. Nat Rev Mol Cell Biol. (2008) 9:367-77. doi: 10.1038/nrm2391

50. Stoner SC. Management of serious cardiac adverse effects of antipsychotic medications. Mental Health Clin. (2017) 7:24654. doi: $10.9740 / \mathrm{mhc} .2017 .11 .246$

51. Gonçalves P, Araújo JR, Martel F. Antipsychotics-induced metabolic alterations: focus on adipose tissue and molecular mechanisms. Euro Neuropsychopharmacol. (2015) 25:1-16. doi: 10.1016/j.euroneuro.2014.11.008

52. Ma W, Huang T, Zheng Y, Wang M, Bray GA, Sacks FM, et al. Weightloss diets, adiponectin, and changes in cardiometabolic risk in the 2year POUNDS lost trial. J Clin Endocrinol Metab. (2016) 101:241522. doi: 10.1210/jc.2016-1207

Conflict of Interest: OA has received speaker's honorarium from Lundbeck and is a consultant for HealhLytix.

The remaining authors declare that the research was conducted in the absence of any commercial or financial relationships that could be construed as a potential conflict of interest.

Copyright $\odot 2021$ Reponen, Tesli, Dieset, Steen, Vedal, Szabo, Werner, Lunding, Johansen, Rødevand, Andreassen and Ueland. This is an open-access article distributed under the terms of the Creative Commons Attribution License (CC BY). The use, distribution or reproduction in other forums is permitted, provided the original author(s) and the copyright owner(s) are credited and that the original publication in this journal is cited, in accordance with accepted academic practice. No use, distribution or reproduction is permitted which does not comply with these terms. 